Research Article

\title{
Impacts of Urbanization on Extreme Regional Precipitation Events
}

\author{
Wei Wu $\left(\mathbb{D}^{1},{ }^{1}\right.$ Lei Ren $\mathbb{D}^{1},{ }^{1}$ Yingjian Wei, ${ }^{2}$ and Mengjing Guo ${ }^{1}$ \\ ${ }^{1}$ State Key Laboratory of Eco-hydraulics in Northwest Arid Region of China, Xi'an University of Technology, Xi'an 710048, China \\ ${ }^{2}$ Power China Kunming Engineering Corporation Limited, Kunming 650051, China \\ Correspondence should be addressed to Lei Ren; 543491057@qq.com
}

Received 18 June 2021; Accepted 4 August 2021; Published 12 August 2021

Academic Editor: Daqing Gong

Copyright $(02021$ Wei Wu et al. This is an open access article distributed under the Creative Commons Attribution License, which permits unrestricted use, distribution, and reproduction in any medium, provided the original work is properly cited.

Urbanization significantly impacts regional precipitation. In this study, using the city of Xi'an in China as a study area, we analyze the variation in land use and the characteristics of the variation in extreme precipitation events and discuss the response of urbanization to extreme precipitation. The main conclusions drawn are as follows. (1) The construction land area of the study area rapidly expanded in size from $80.76 \mathrm{~km}^{2}$ in 1984 to $400.3 \mathrm{~km}^{2}$ in 2018 . The transfer of land use mainly occurred as the transfer from farmland to other types of land use. The urban land use intensity continues to increase. (2) The intensities and different frequency indices of extreme precipitations both show an increasing trend. In addition, the former exhibits periodic alternation with a dominant frequency of 21 years during the flood season, and both the fluctuation amplitude and maximum value of the periodic variation increase with time. (3) The urbanization in the study area has a significant impact on $\mathrm{Rx} 1 \mathrm{~h}$ and $\mathrm{Rx} 2 \mathrm{~h}$ precipitations, but little impact on the intensity of long-duration precipitation. Hence, urbanization significantly increases the intensity of extreme precipitation. After the 2000s, the urban building area, rainfall frequency, and rainfall intensity growth trends are highly consistent.

\section{Introduction}

Urbanization is rapidly occurring across the world, and the global urban population is predicted to increase by 2.5 billion by 2050 [1]. Increasing urban population can directly lead to enlarged urban construction areas and changes in land use types, which can further alter the physical properties of the underlying urban surface and greatly affect the characteristics of regional hydrological circulation [2, 3]. Additionally, given the clustering trends of population and industry, different artificial heat sources, carbon sources, and pollutant emissions directly affect the chemical and physical properties of the atmosphere, which can lead to changes in the movement state of near-surface matter and energy in local urban areas and over an even larger range and have complex effects on a series of meteorological and hydrological elements, including wind fields, temperature, precipitation, evapotranspiration, and runoff. The urban interior is characterized by low water content and low latent heat, and its surface heat is released mainly in the form of sensible heat. In addition, large quantities of heat are released as a result of high energy consumption (e.g., air conditioning and transportation). As a result, the urban surface temperature is remarkably higher than that of the corresponding underlying natural surface $[4,5]$. These factors make precipitation more likely to occur in urban areas, known as the rain island effect $[6,7]$.

Thunderstorms are more likely to occur in urban areas than in suburban areas $[8,9]$. The mechanisms of the impact of urbanization on precipitation mainly include the urban heat island effect, underlying surface changes, and aerosol emissions. Heat island circulation weakens the stability of the urban atmosphere, which significantly promotes the formation of convective weathers such as thunderstorms, heavy precipitation, and strong storms, and changes the dynamic structures of clouds and storms, leading to extreme events of enhanced intensity as a result of the strengthened convection in downwind urban areas [10]. In addition, the gravitational 
waves induced by the urban heat island effect can cause the airflow to ascend in downwind urban areas, which can easily trigger and strengthen moist convection, causing extreme precipitation events in some urban and downwind areas [11]. Changes in the underlying urban surface can reduce the nearsurface air humidity, which further leads to decreases in evapotranspiration and precipitation in summer [12]. Aerosols such as smoke dust over urban areas are also conducive to the formation of clouds and thus rain [13,14], but the impact of aerosols on precipitation is influenced by many factors such as climate and geography and thus is highly uncertain [15-18].

The impact of urbanization on precipitation can be assessed by two methods: data analysis and numerical simulation [19]. The data analysis method compares the different precipitation elements in the urban area and the adjacent suburban or rural areas at different positions and uses the obtained difference as the main index for assessing the level of the impact of urbanization on precipitation. This method falls into two categories: station data analysis and integrated remote sensing data analysis. The former requires the use of a series of long-duration precipitation observations in both urban and rural areas and is limited by its poor spatial coverage. In comparison, the latter can overcome the limit on spatial coverage and improve the construction of precipitation samples in both urban and background areas $[20,21]$. To investigate the impact of urbanization on precipitation, the numerical simulation method focuses on the simulation of single-factor impacts and integrated impacts. In the single-factor impact simulation, the impacts of the urban heat island, the underlying surface, and the aerosols are separately simulated and assessed. The most direct approach for the integrated impact simulation is to conduct control experiments with and without cities or with different scenarios of urban development [22-24]. In the numerical simulation method, sensitivity analysis can be carried out by varying the model parameters to determine the impacts of different factors in the urbanization process. However, numerical simulation results are usually of low accuracy [24]. In contrast, the statistical data analysis method can more accurately and directly assess the variation patterns of urbanization and precipitation.

Urbanization can alter the underlying surface properties and the regional precipitation, both of which often lead to serious urban waterlogging problems $[25,26]$. Thus, it is of great importance to understand the impact of urbanization on precipitation $[27,28]$. Existing studies on this subject mostly focus on analyzing daily precipitation data to obtain the trends of precipitation, while there is little research on extreme precipitation events of short duration, which are more likely to cause urban waterlogging $[29,30]$. To address this knowledge gap, the spatiotemporal patterns of urban sprawl in the central urban area are investigated in the present study based on the interpretation of remote sensing data, taking the city of Xi'an in China as a study area. In addition, the pattern of the maximum of short-duration precipitation in flood season is analyzed using the shortduration rainfall data of the urban area. Finally, the impact of urbanization on extreme precipitation events is discussed through comparison.

\section{Overview of the Study Area}

In this study, we chose the city of Xi'an in China as our study area (Figure 1). Xi'an is located in central China between $108^{\circ} 46^{\prime}-109^{\circ} 5^{\prime} \mathrm{E}$ and $34^{\circ} 11^{\prime}-34^{\circ} 22^{\prime} \mathrm{N}$, with a total area of $10,100 \mathrm{~m}^{2}$. The study area is in a warm-temperature zone with a semihumid continental monsoonal climate. It is characterized by well-delineated seasons regarding humidity and temperature and by unevenly distributed seasonal precipitation. More than $80 \%$ of the precipitation occurs during flood season, and the amount of precipitation from July to September accounts for more than half of the annual precipitation. In recent years, heavy, short-duration precipitation has occurred multiple times and resulted in severe urban waterlogging. The study area has experienced rapid urbanization. As a result, the original natural vegetation and farmlands are now covered by impermeable surfaces, resulting in significant changes to the underlying surface. Currently, the types of urban land use mainly include residential lands, commercial lands, parklands, and education and research lands, which can fully reflect the urbanization process.

\section{Data and Methodology}

3.1. Data. The land-use data of the study area are derived from the Geospatial Data Cloud platform maintained by the China Academy of Sciences Computer Network Information Center (http://www.gscloud.cn) as well as from Google Earth. The LANDSAT remote sensing image data of 1985, $1990,1995,2000,2005,2010,2015$, and 2018, a span of 34 years, which could represent the degree of urbanization of the study area in different periods are selected. The resolution of the data is $30 \mathrm{~m} \times 30 \mathrm{~m}$. The image data were taken during April to August with the cloudiness controlled to within $5 \%$.

The hourly precipitation data of 1982 to 2018 of the Xi'an station (S1) and Huxian station (S2) in the study area are selected. Since the surrounding regions of the S1 station experienced urbanization after the 1980s, station S1 is used as an urban precipitation observation station in the present study. The long-term variation in precipitation at this station can be viewed as a representation of the local impact of urbanization on rainfall and the background fluctuations in the regional climate. Thus, the precipitation data at station S1 are selected to analyze the impact of urbanization on the evolution of extreme precipitation events. In contrast, station S2 is located in a rural area that is barely impacted by urbanization. Thus, the precipitation variation at station S2 is considered to represent the natural variation due to climate change.

\subsection{Methodology}

3.2.1. Urbanization of Study Area. In this study, the different types of land-use data are categorized by using the ENVI (Environment for Visualizing Images) software. Then, the accuracy of the calculation results is evaluated to ensure that the accuracy is greater than $80 \%$ with a kappa coefficient 


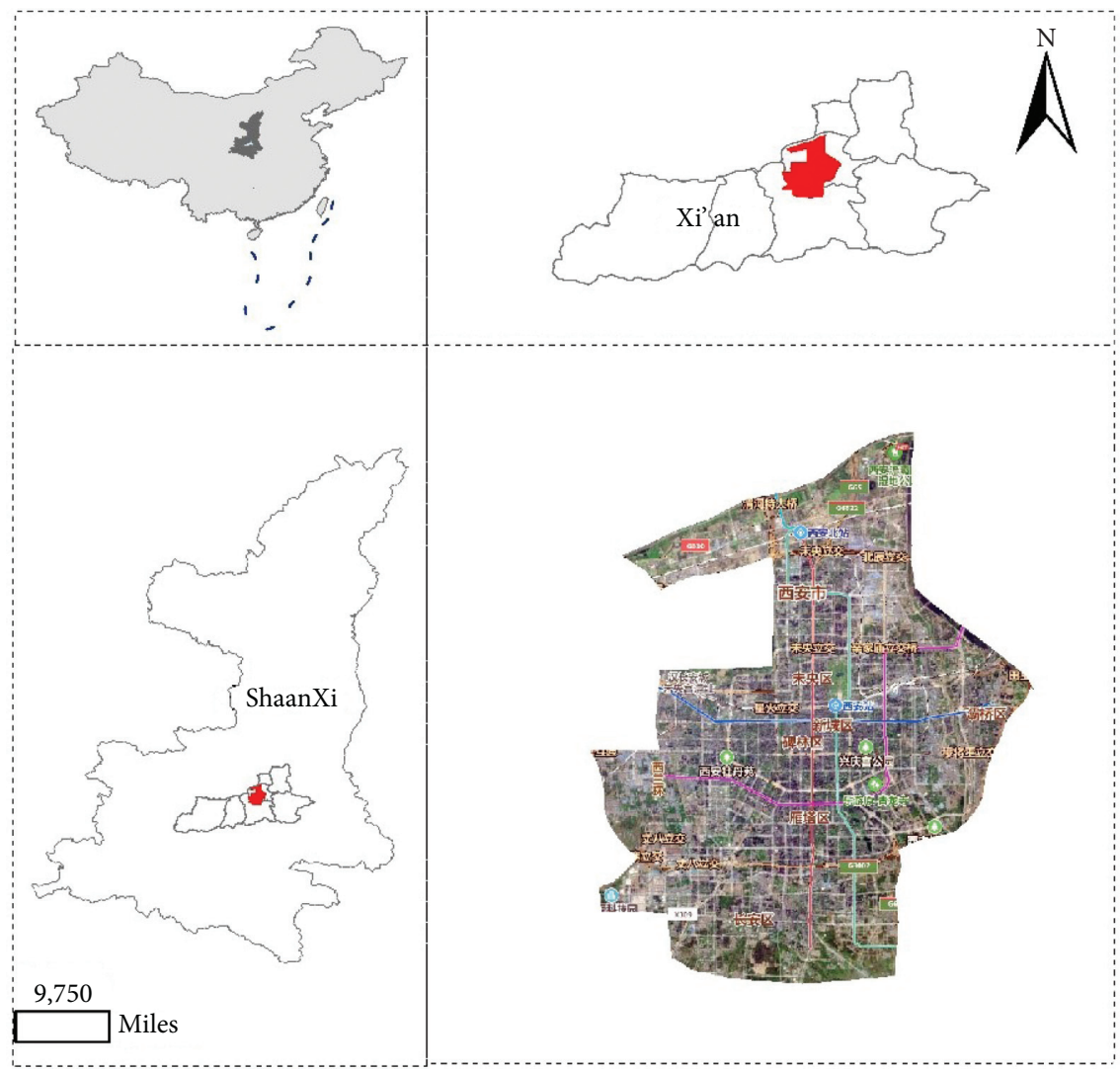

Figure 1: Schematic diagram of the study area.

greater than 0.8 . The land use within the study area is categorized into five types, namely, farmland, vegetation, bare land, water body, and construction land. The spatiotemporal evolution of the urban sprawl in the study is mainly analyzed in terms of the speed, direction, and intensity of the changes in urban land use.

Land use intensity can reflect both the depth and breadth of urban land use and the intensity and development stages of urbanization. In fact, increasing land use intensity is a process during which the natural attributes of land are continuously changed as a result of human activities. There exist differences in the intensities of different land use types. In this study, the land use intensity is rated with a four-level scale based on the natural equilibrium state of the natural land complex under the influence of social factors and assigned an index according to the rated level [31]. The calculation equation is

$$
\begin{aligned}
& L=100 \times \sum_{I=1}^{4} \frac{A_{I} \times S_{i}}{S}, \\
& L \in[100,400], \quad i=1,2,3,4,
\end{aligned}
$$

where $L$ is the composite index for the land use intensity of an area, $A_{I}$ is the rating index of the intensity of the level $i$ land use in the area, $S_{i}$ is the area of the level $i$ land use in the area, and $S$ is the total area of the land in the area.

The spatial autocorrelation analysis method (Moran's index method) is used to analyze the spatial correlations of land use intensities. That is, in the analysis of the spatial correlations of land use intensities, Moran's index is calculated to determine the spatial clustering of the land use intensities. Moran's index $I$ is in the range of $[-1,1] . I<0$, $I=0$, and $I>0$ indicate negative, no, and positive correlations, respectively. The greater the $I$ value is, the higher the spatial correlation and the clustering.

3.2.2. Extreme Precipitation in the Study Area. In this study, the extreme precipitation events are assessed in terms of intensity and occurrence frequency. The indices for the extreme precipitation intensity include the maximum 1hour precipitation intensity during flood season ( $\mathrm{Rx} 1 \mathrm{~h})$, the maximum 2-hour precipitation intensity during flood season ( $\mathrm{Rx} 2 \mathrm{~h})$, the maximum 3-hour precipitation intensity during flood season ( $\mathrm{Rx} 3 \mathrm{~h})$, the maximum 6-hour precipitation intensity during flood season $(\mathrm{Rx} 6 \mathrm{~h})$, the maximum 12-hour precipitation intensity during flood season ( $\mathrm{Rx} 12 \mathrm{~h})$, and the maximum 24-hour precipitation intensity during flood season $(\mathrm{Rx} 24 \mathrm{~h})$, based on which a series of extreme precipitation intensities of varying durations during flood season is selected using the annual maximum method. The indices of the extreme precipitation frequency mainly include the number of heavy precipitation hours, the number of heavy-rain days, and the number of rainstorm days. The percentile threshold method is used to select the 99-percentile hourly precipitation as the threshold value for the hourly heavy precipitation. In addition, the fixed-value 
method is employed to choose the rainstorm and heavy-rain values as the threshold values for calculating the numbers of rainstorm days and heavy-rain days, respectively. The temporal series of the occurrence frequencies of extreme precipitation events is also selected (Table 1). The tendency and abrupt change of the extreme precipitation events are analyzed using the Mann-Kendall (MK) nonparametric statistical test, and the periodic variations in extreme precipitation events are examined using the wavelet analysis method.

\section{Results}

4.1. Land-Use Distribution in Central Urban Area and Its Variation. Based on the interpretation of eight sets of remote sensing image data in the central urban area from 1984 to 2018, the area variation map (Figure 2) and the spatial distribution variation map (Figure 3 ) for the land use in the urban area are obtained.

The results show that the farmland area in the central urban area of the study area varies the most significantly. Specifically, this area decreased from $441.05 \mathrm{~km}^{2}$ in 1984 to $6.18 \mathrm{~km}^{2}$ in 2018 , and the corresponding percentage decreased from $81.81 \%$ to $1.15 \%$. In particular, farmland showed the largest decrease from 2009 to 2015, with an area decrease of $148.05 \mathrm{~km}^{2}$, accounting for $27.46 \%$ of the total area. In comparison, the area of construction land increased significantly from $80.76 \mathrm{~km}^{2}$ in 1984 to $400.03 \mathrm{~km}^{2}$ in 2018 , with the corresponding percentage increasing from $14.98 \%$ to $74.25 \%$. Additionally, the construction land area increased the most significantly from 2009 to 2015, with an increase of $105.36 \mathrm{~km}^{2}$ or $19.54 \%$ of the total area.

4.2. Trends of Precipitation during Flood Season. The MK nonparametric statistical test method is used to calculate the trends of the intensities and occurrence frequencies of extreme precipitation with different durations in the study area (Table 2). The intensities of the extreme precipitations with different durations in the study area all exhibit an increasing trend. The $\mathrm{Z}$-scores of the increases in the extreme precipitation intensities $\mathrm{Rx} 1 \mathrm{~h}, \mathrm{Rx} 12 \mathrm{~h}$, and $\mathrm{Rx} 24 \mathrm{~h}$ are all greater than 1.28 and pass the $90 \%$ significance test, and the corresponding nonparametric Sen's slopes are 1.06, 1.64, and $2.16 \mathrm{~mm} / 10 \mathrm{a}$, respectively. The descending order of the significance levels of the trends of the intensities of extreme precipitation with different durations is $\mathrm{Rx} 12 \mathrm{~h}>$ $\mathrm{Rx} 24 \mathrm{~h}>\mathrm{Rx} 1 \mathrm{~h}>\mathrm{Rx} 2 \mathrm{~h}>\mathrm{Rx} 6 \mathrm{~h}>\mathrm{Rx} 3 \mathrm{~h}$. The descending order of the increases in the calculated intensities of precipitation with different durations is $\mathrm{Rx} 24 \mathrm{~h}>\mathrm{Rx} 12 \mathrm{~h}>\mathrm{Rx} 1 \mathrm{~h}>$ $\mathrm{Rx} 2 \mathrm{~h}>\mathrm{Rx} 6 \mathrm{~h}>\mathrm{Rx} 3 \mathrm{~h}$. In addition, different extreme precipitation frequency indices exhibit increasing trends; the number of 99-percentile heavy precipitation hours shows a significant increasing trend with a $Z$-score of 1.79 , which passes the $95 \%$ significance test with an increase of $0.29 \mathrm{~h} /$ $10 \mathrm{a}$.

The long-term variations in the intensity of extreme precipitation in the study area are statistically analyzed (Figure 4). The results show that the slopes of the fitted trend lines for the intensities of extreme precipitation with different durations are all positive, showing an increasing trend with fluctuations. The fluctuation peak continues to increase, while the fluctuation valley does not vary significantly. Before the 1980s, the intensities of extreme precipitation with different durations were in a steady state with a small fluctuation range. After the 1980s, the fluctuations gradually intensified with an increasing difference in annual precipitation.

\section{Discussion}

\subsection{Variations in Urban Land Use}

5.1.1. Variation Direction of Land Use in Central Urban Area. The transformation matrix for the land use during different periods is calculated by using the software ENVI, and then the variation direction of land use is analyzed. The land-use transformation matrices for the early period (1990) and the late period (2018), as given in Table 3, show that the transformation of land use in the central urban area of the study area mainly involves the transfer of farmland to other types of land use, while the transfers between other types of land use are not significant.

From 1990 to 2018, the farmland in the study area continuously decreased, with the proportion of its area decreasing from $81.81 \%$ to $1.15 \%$. The farmland was mainly transferred to construction land and vegetation, with transferred areas of $234.27 \mathrm{~km}^{2}$ and $84.71 \mathrm{~km}^{2}$, respectively. Construction land was mainly transferred to vegetation with an area of $16.44 \mathrm{~km}^{2}$ that was probably related to human activities such as newly constructed park green spaces and urban greening. Vegetation was mainly transferred to construction land with an area of $43.14 \mathrm{~km}^{2}$, while the areas of water bodies and bare land did not vary significantly.

In general, the different types of land use in the central urban area of the study area all experienced transformation. The descending order of the types of areas that were converted to a different type of land use is farmland $\left(327.53 \mathrm{~km}^{2}\right)>$ vegetation $\left(45.06 \mathrm{~km}^{2}\right)>$ construction land $\left(18.74 \mathrm{~km}^{2}\right)>$ water bodies $\left(7.66 \mathrm{~km}^{2}\right)>$ bare land $\left(0.59 \mathrm{~km}^{2}\right)$. The descending order of the types of land use that areas were converted to is construction land $\left(283.23 \mathrm{~km}^{2}\right)>$ vegetation $\left(103.2 \mathrm{~km}^{2}\right)>$ bare land $\left(9.1 \mathrm{~km}^{2}\right)$ $>$ water bodies $\left(3.26 \mathrm{~km}^{2}\right)>$ farmland $\left(0.91 \mathrm{~km}^{2}\right)$.

5.1.2. Characteristics of the Spatiotemporal Distribution of Land Use in the Central Urban Area. The intensity of land use reflects the breadth and depth of land use. A low intensity of land use in a region indicates a small impact from human activities on the natural environment in this region, while a high intensity of land use mirrors the impact of human activities on the natural attributes of the land, resulting in changes in the physical properties of the underlying surface.

The central urban area of the study area is divided into grids with equal spacing using the software ArcGIS, resulting in a total of 2280 grids with a spatial resolution of $500 \mathrm{~m} \times 500 \mathrm{~m}$. Within each grid, the land use intensities are 
TABLE 1: Indices for extreme precipitation events and their definitions.

\begin{tabular}{|c|c|c|c|c|}
\hline Extreme precipitation index & Name & Symbol & Unit & Definition \\
\hline \multirow{6}{*}{ Extreme precipitation intensity } & $\begin{array}{l}\text { Maximum 1-hour precipitation } \\
\text { intensity during flood season }\end{array}$ & Rx1h & $\mathrm{mm}$ & $\begin{array}{c}\text { Maximum annual 1-hour precipitation during } \\
\text { flood season }\end{array}$ \\
\hline & $\begin{array}{l}\text { Maximum 2-hour precipitation } \\
\text { intensity during flood season }\end{array}$ & $\mathrm{Rx} 2 \mathrm{~h}$ & $\mathrm{~mm}$ & $\begin{array}{c}\text { Maximum annual 2-hour precipitation during } \\
\text { flood season }\end{array}$ \\
\hline & $\begin{array}{l}\text { Maximum 3-hour precipitation } \\
\text { intensity during flood season }\end{array}$ & $\mathrm{Rx} 3 \mathrm{~h}$ & $\mathrm{~mm}$ & $\begin{array}{c}\text { Maximum annual 3-hour precipitation during } \\
\text { flood season }\end{array}$ \\
\hline & $\begin{array}{l}\text { Maximum 6-hour precipitation } \\
\text { intensity during flood season }\end{array}$ & Rx6h & $\mathrm{mm}$ & $\begin{array}{c}\text { Maximum annual 6-hour precipitation during } \\
\text { flood season }\end{array}$ \\
\hline & $\begin{array}{l}\text { Maximum 12-hour precipitation } \\
\text { intensity during flood season }\end{array}$ & $\mathrm{Rx} 12 \mathrm{~h}$ & $\mathrm{~mm}$ & $\begin{array}{c}\text { Maximum annual 12-hour precipitation during } \\
\text { flood season }\end{array}$ \\
\hline & $\begin{array}{l}\text { Maximum 24-hour precipitation } \\
\text { intensity during flood season }\end{array}$ & $\mathrm{Rx} 24 \mathrm{~h}$ & $\mathrm{~mm}$ & $\begin{array}{c}\text { Maximum annual 24-hour precipitation during } \\
\text { flood season }\end{array}$ \\
\hline \multirow{3}{*}{ Extreme precipitation frequency } & Number of heavy precipitation hours & R99p & $\mathrm{h}$ & $\begin{array}{l}\text { Number of hours during which hourly } \\
\text { precipitation is greater than the 99-percentile } \\
\text { threshold value }\end{array}$ \\
\hline & Number of heavy-rain days & $\mathrm{R} 25 \mathrm{~mm}$ & $\mathrm{~d}$ & $\begin{array}{c}\text { Number of days during which daily precipitation } \\
\text { is greater than } 25 \mathrm{~mm}\end{array}$ \\
\hline & Number of rainstorm days & $\mathrm{R} 50 \mathrm{~mm}$ & $\mathrm{~d}$ & $\begin{array}{c}\text { Number of days during which daily precipitation } \\
\text { is greater than } 50 \mathrm{~mm}\end{array}$ \\
\hline
\end{tabular}

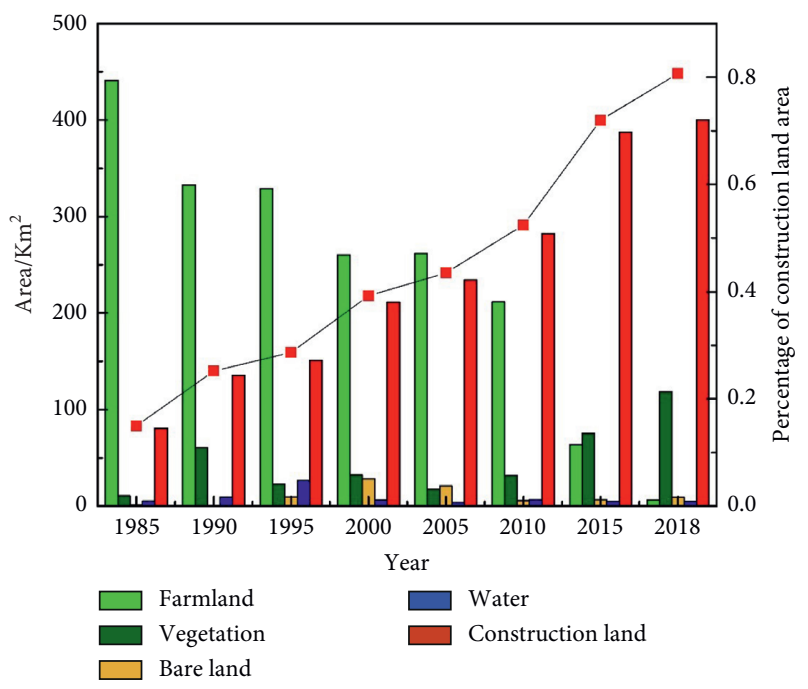

Figure 2: Variations in land use and coverage area in the central urban area of the study area.

calculated, and a spatial interpolation analysis is conducted on the varied intensities of land use in different periods by the Kriging interpolation method, from which the spatial distribution of the land use intensities in the central urban area of the study area during different periods is obtained (Figure 5).

The results show that the expansion of regions with highintensity land use in the central urban area of the study area is consistent with the variation in the area of construction land. At the beginning of the study period, the high-value regions were mainly clustered in the old town with a planar distribution pattern. Due to the impact of urbanization, these high-value regions started to expand with the construction land toward the south and north until the entire area was covered, exhibiting a multipoint expansion pattern.
In the low-value regions, the types of land use include farmland, vegetation, water bodies, and bare land. The spatial shrinkage of the distributed area of the low-value regions is mainly related to the decrease in the farmland area and the increase in the construction land area. At the initial stage of the study period, the low-value regions were mainly distributed in the suburban area. As urbanization progressed, the construction land encroached on the farmland and vegetation and, as a result, the land use intensity of the suburban area increased.

The Moran's index method is used to analyze the spatial correlation model of land use intensities in the central urban area of the study area from 1984 to 2018 (Figure 6). The calculation results show that the Moran's indices of the land use intensities are all greater than zero from 1984 to 2018, 


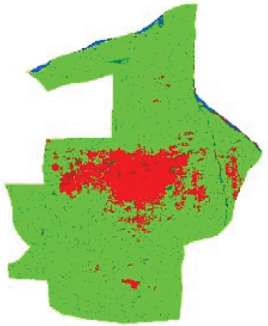

1985

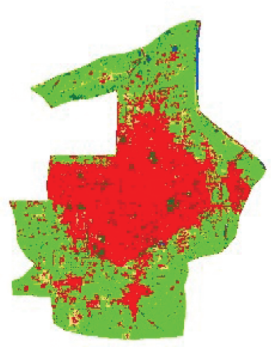

2005

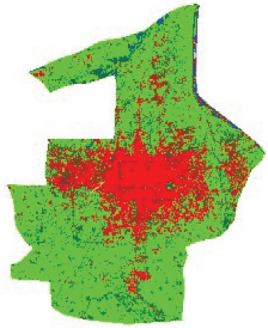

1990

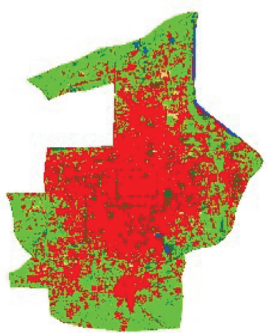

2010

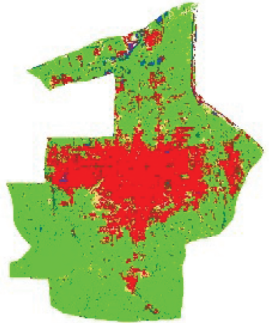

1995

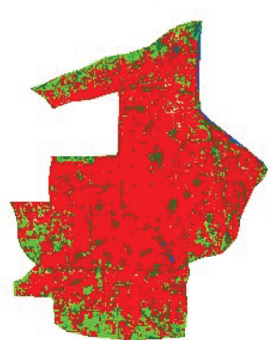

2015

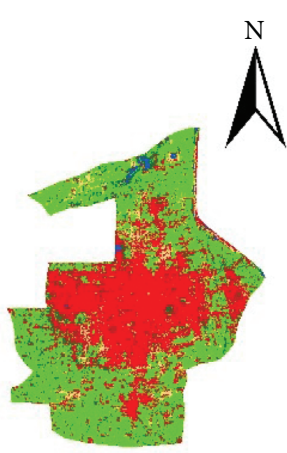

2000

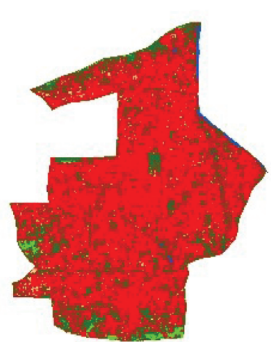

2018

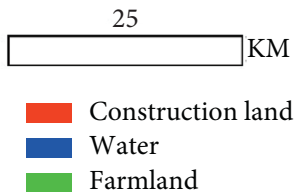

KM

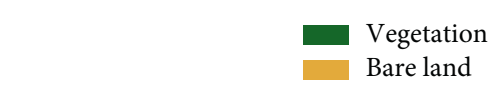

FIgURE 3: Spatial distribution of land use in the central urban area of the study area from 1984 to 2018.

TABLE 2: MK tests and slopes of indices of extreme precipitation in the central urban area of the study area.

\begin{tabular}{lccc}
\hline Extreme precipitation index & Z-score & Variation magnitude of nonparametric Sen's & Confidence level (\%) \\
\hline Rx1h & 1.29 & $1.06(\mathrm{~mm} / 10 \mathrm{a})$ & $>90$ \\
Rx2h & 1.02 & $0.95(\mathrm{~mm} / 10 \mathrm{a})$ & $<90$ \\
Rx3h & 0.63 & $0.56(\mathrm{~mm} / 10 \mathrm{a})$ & $<90$ \\
Rx6h & 0.99 & $0.80(\mathrm{~mm} / 10 \mathrm{a})$ & $<90$ \\
Rx12h & 1.53 & $1.63(\mathrm{~mm} / 10 \mathrm{a})$ & $>90$ \\
Rx24h & 1.51 & $2.16(\mathrm{~mm} / 10 \mathrm{a})$ & $>90$ \\
R25mm & 1.05 & $0(\mathrm{~d} / 10 \mathrm{a})$ & $<90$ \\
R50mm & 0.64 & $0(\mathrm{~d} / 10 \mathrm{a})$ & $<90$ \\
R99p & 1.79 & $0.29(\mathrm{~h} / 10 \mathrm{a})$ & $>95$ \\
\hline
\end{tabular}

indicating that the land use intensities show an overall positive correlation and have a clustered spatial distribution tendency. However, the Moran's index gradually decreases with time, indicating that with the multipoint expansion of construction land, the clustering of land use intensities in the study area decreases. Therefore, it is concluded that urban sprawl has impacted the spatial pattern of the land use intensities in the urban area.

Statistical analysis on the land use intensities in the central urban area of the study area from 1984 to 2018 is performed (Figure 7). The statistical results show the following. The overall average of the land use intensities is in the range of 300 to 360 . The land use intensities in the central urban area increased from 1984 to 2015 and started to decrease from 2015 to 2018 , which may be attributed to the increase in vegetation area. The standard deviation can reflect the dispersion of land use intensities. The standard deviation of the land use intensities exhibited an increasing trend from 1984 to 2005, decreased from 2005 and 2009, and has been gradually increasing since 2009 . This reflects a change in the pattern of urban expansion. The early stage of urbanization mainly included the transfer of farmland and vegetation to construction land, with most of the land transforming from low land use intensities to high land use intensities. In comparison, the late stage of urbanization mainly included transformation from farmland to construction land and vegetation, and the role of urban greening and parks in the course of 

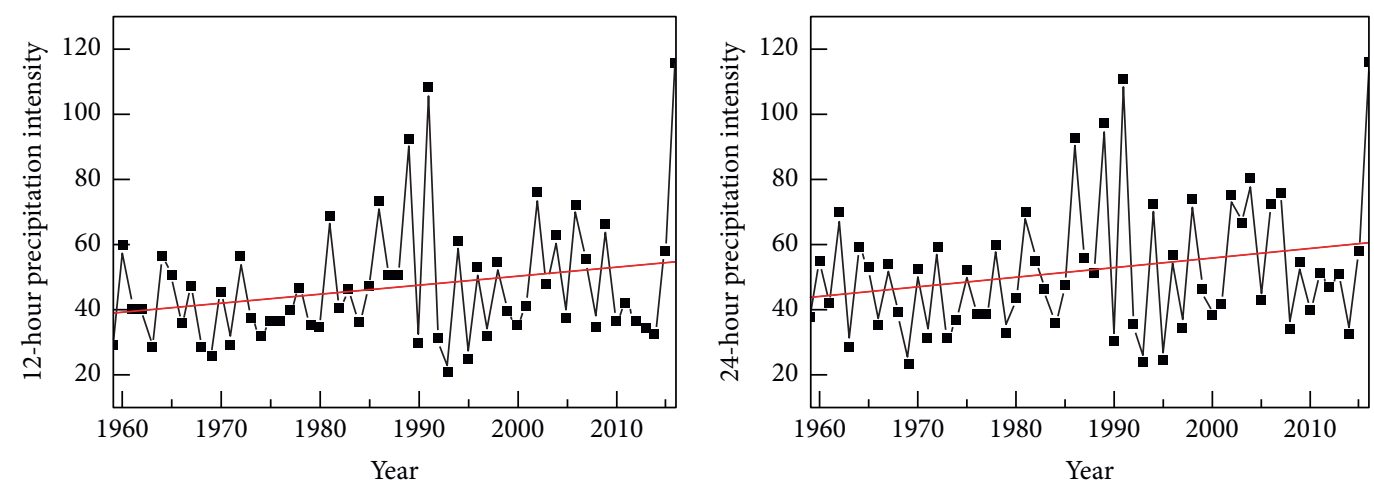

- Trendline

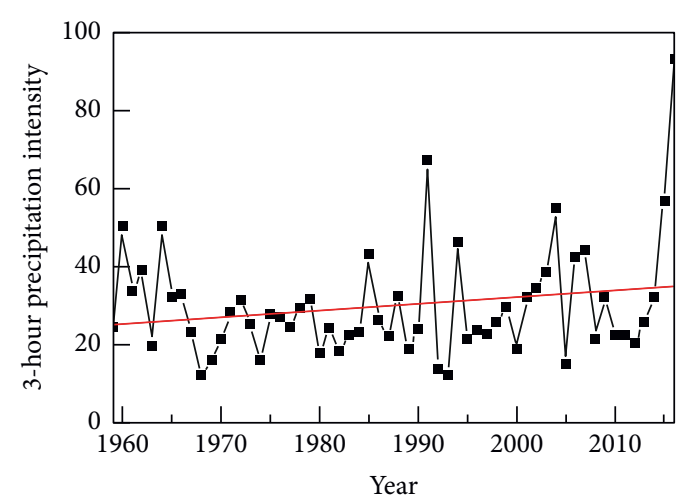

— Trendline

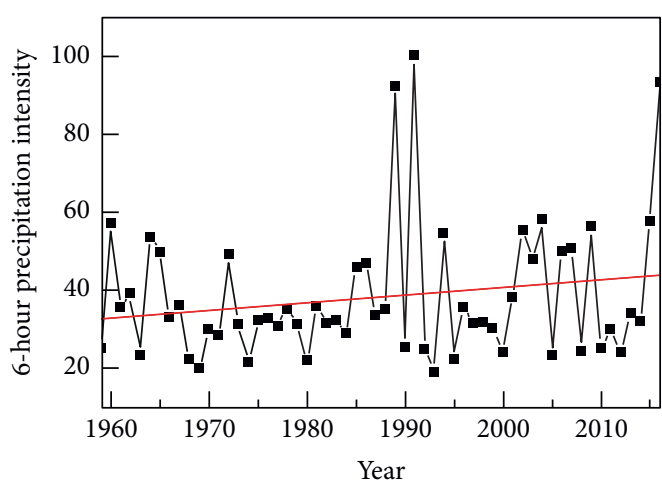

_ Trendline
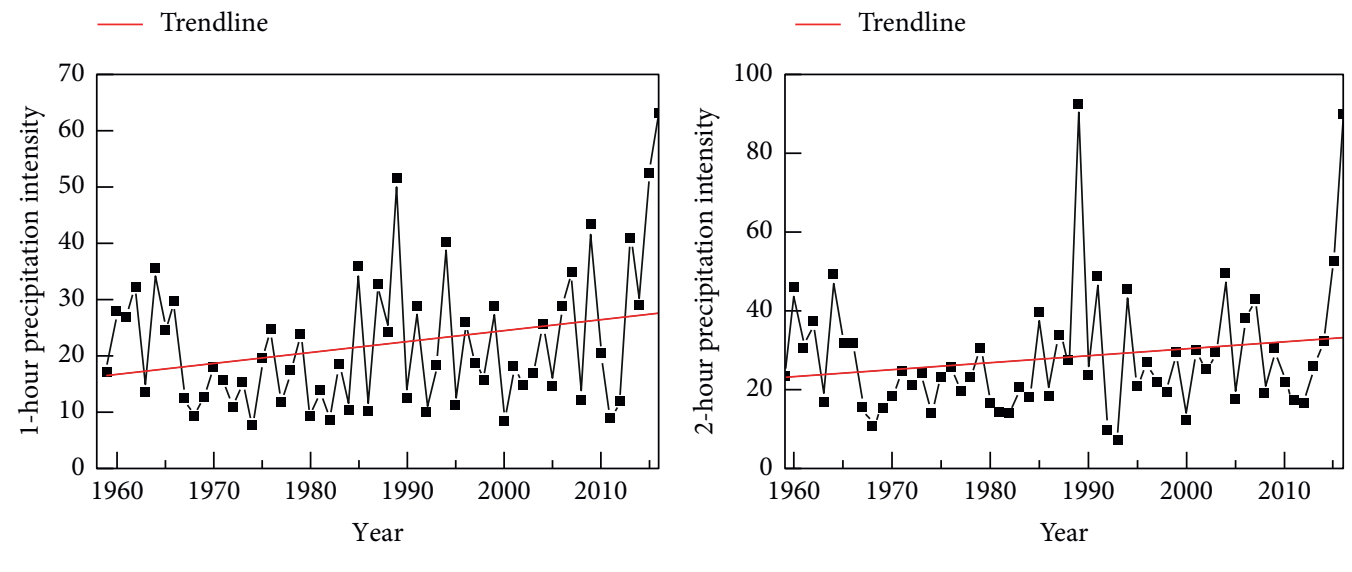

— Trendline

— Trendline

FIGURE 4: Trends of intensities of extreme precipitation with different durations.

urbanization started gaining attention. This transfer reflects the transformation from low to low/medium land use intensities.

To further analyze the characteristics of the land use intensities and their variations over different periods, the land use intensities are classified into the following levels: low-intensity land use $(L \leq 200)$, low/medium-intensity land use $(200<L \leq 250)$, medium-intensity land use $(250<L \leq 300)$, medium/high-intensity land use $(300<L$ $\leq 350)$, and high-intensity land use $(350<L \leq 400)$. The proportions of the different land use intensity levels in different periods are calculated and presented in Figure 8 using histograms in terms of frequency counts. The results show the following. The proportion of high-intensity land use increased each year, with an increasing trend consistent with the variation in construction land. The proportions of medium/high-intensity and medium-intensity land fluctuated and decreased with time. The variations in the other levels of land use intensities were not pronounced. The land use intensities in the central urban area of the study area varied considerably in different periods. That is, the land use intensities were dominated by medium/high-intensity land use from 1984 to 2005 and by high-intensity land use from 2005 to 2018, thus reflecting the changes in the dominant types of land use in different periods. Overall, the land use intensities of the entire study area transformed with time from low-intensity to highintensity land use. 
TABLE 3: Transformation matrix of land use in the central urban area of the study area from 1990 to 2018.

\begin{tabular}{|c|c|c|c|c|c|c|c|}
\hline \multirow{2}{*}{ Land use type } & \multirow{2}{*}{1990} & \multicolumn{6}{|c|}{2018} \\
\hline & & Construction land & Water bodies & Bare land & Farmland & Vegetation & Total \\
\hline \multirow{3}{*}{ Construction land } & Area & 116.98 & 0.90 & 1.27 & 0.14 & 16.44 & \multirow{3}{*}{135.72} \\
\hline & Transfer rate (positive) & 86.19 & 0.66 & 0.94 & 0.10 & 12.11 & \\
\hline & Transfer rate (negative) & 29.24 & 17.36 & 13.97 & 2.20 & 13.86 & \\
\hline \multirow{3}{*}{ Water body } & Area & 5.43 & 1.93 & 0.12 & 0.08 & 2.04 & \multirow{3}{*}{9.59} \\
\hline & Transfer rate (positive) & 56.60 & 20.07 & 1.24 & 0.84 & 21.26 & \\
\hline & Transfer rate (negative) & 1.36 & 37.12 & 1.30 & 1.30 & 1.72 & \\
\hline \multirow{3}{*}{ Bare land } & Area & 0.40 & 0.11 & 0.01 & 0.00 & 0.08 & \multirow{3}{*}{0.60} \\
\hline & Transfer rate (positive) & 66.87 & 18.21 & 1.79 & 0.00 & 13.13 & \\
\hline & Transfer rate (negative) & 0.10 & 2.12 & 0.12 & 0.00 & 0.07 & \\
\hline \multirow{3}{*}{ Farmland } & Area & 234.17 & 1.86 & 6.87 & 5.27 & 84.71 & \multirow{3}{*}{332.80} \\
\hline & Transfer rate (positive) & 70.36 & 0.56 & 2.06 & 1.58 & 25.45 & \\
\hline & Transfer rate (negative) & 58.54 & 35.90 & 75.35 & 85.19 & 71.41 & \\
\hline \multirow{4}{*}{ Vegetation } & Area & 43.14 & 0.39 & 0.84 & 0.69 & 15.36 & \multirow{4}{*}{60.43} \\
\hline & Transfer rate (positive) & 57.05 & 0.52 & 1.12 & 0.91 & 20.31 & \\
\hline & Transfer rate (negative) & 10.79 & 7.51 & 9.26 & 11.18 & 12.95 & \\
\hline & Total & 400.02 & 5.19 & 9.11 & 6.18 & 118.62 & \\
\hline
\end{tabular}
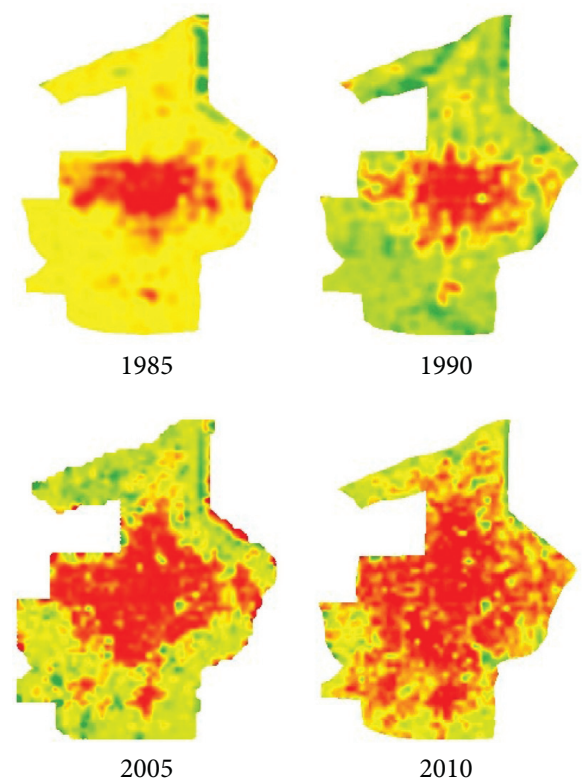

2010

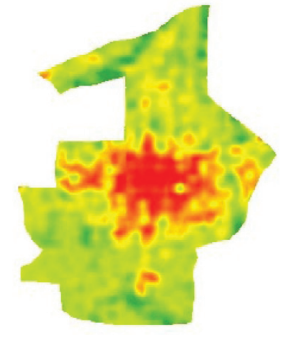

1990

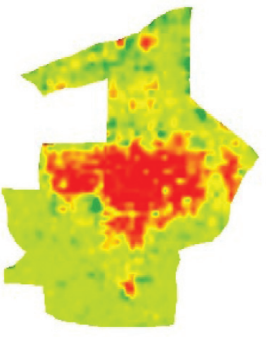

1995
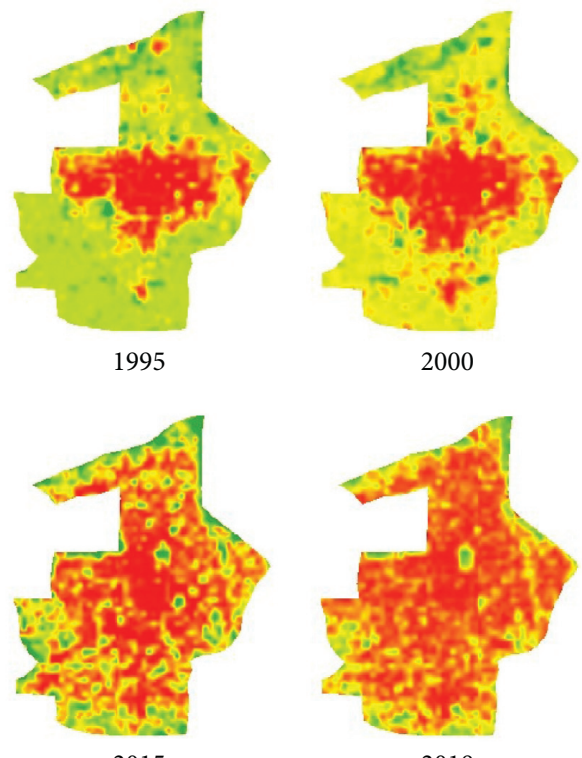

015

VALUE

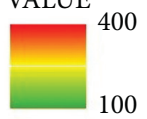

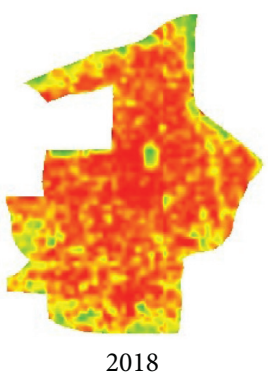

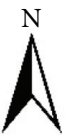

Figure 5: Spatial distribution of land use intensities in the central urban area of the study area from 1984 to 2018.

\subsection{Trends of Intensity and Frequency of Precipitation with Different Durations}

5.2.1. Periodic Characteristics of Intensities of Precipitation with Different Durations during Flood Season. The periodic trends of the intensities of precipitation with different durations during flood season are calculated by wavelet analysis, and the contours of the real parts of the calculated wavelet coefficients are plotted in Figure 9. The wavelet variance is calculated based on the wavelet coefficients
(Figure 10). The results show the following. The evolution processes of extreme precipitation with different durations in the study area mainly exhibit two patterns of periodic variation, that is, a period of 12 to 16 years and a period of 20 to 25 years. The time scale of 12 to 16 years shows strong characteristics of periodic alternations, with a central scale of approximately 15 years. In comparison, the time scale of 20 to 25 years exhibits strong characteristics of positive and negative alternations and significant phase shifts, with a central scale of approximately 21 years. The dominant 


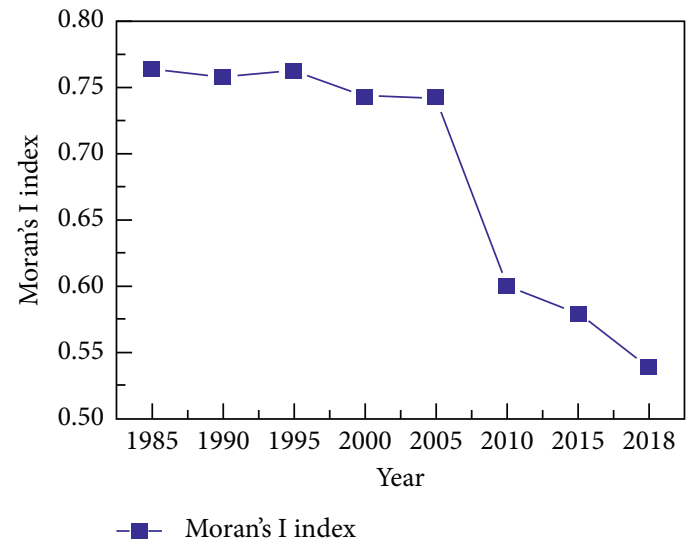

Figure 6: Variation in Moran's index in the central urban area of the study area.

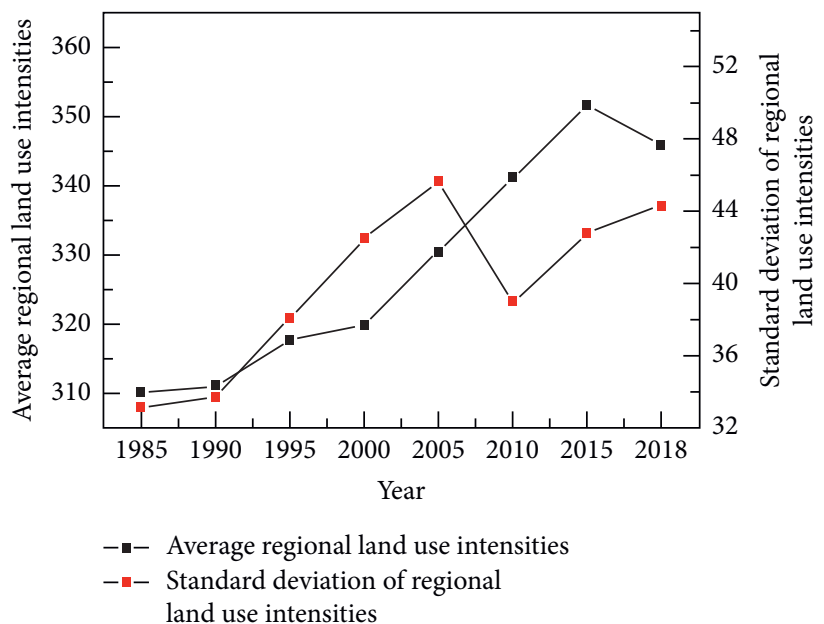

FIgURE 7: Statistics of land use intensities in the central urban area of the study area.

periods of intensities of extreme precipitation with different durations are basically the same, i.e., variation characteristics with a period of 21 years are present. The wavelet coefficients of the dominant periods of the intensities of precipitation with different durations are analyzed, and it is found that the periodic variations intensified with time, exhibiting higher fluctuation magnitudes and larger peaks, which is consistent with the actual fluctuations in the precipitation intensity series.

5.2.2. Abrupt Changes in Intensities of Precipitation with Different Durations during Flood Season. The MK nonparametric test is conducted to analyze the abrupt changes in the intensities of the precipitation with different durations (Figure 11). The results show the following. There were abrupt changes in the intensities of the precipitation with different durations, but the significances of these abrupt changes are relatively low. In particular, the variations in $\mathrm{Rx} 1 \mathrm{~h}$ and $\mathrm{Rx} 2 \mathrm{~h}$ were relatively consistent, and they experienced abrupt changes in 2015, mainly undergoing increases from small-magnitude to large-magnitude fluctuations. The Uf curves further indicate that the intensities of Rx1h and Rx $2 \mathrm{~h}$ have been increasing since the 1980s. Abrupt changes occurred for the Rx3h and Rx6h precipitation intensities in 1960 and 2015, respectively, with a relatively low significance, and the variations before and after the abrupt change in 2015 are consistent with those of $\mathrm{Rx} 1 \mathrm{~h}$ and Rx2h. The Uf curves of Rx3h and Rx6h further show that both indices experienced a process of first steady fluctuation, then increased by small magnitudes, and finally significantly increased. Thus, their overall variation trends are characterized by insignificant increases. $\mathrm{Rx} 12 \mathrm{~h}$ and $\mathrm{Rx} 24 \mathrm{~h}$ experienced multiple insignificant abrupt changes, indicating that there were large fluctuations in the data series during this time period.

5.3. Impacts of Urbanization on Extreme Precipitation. The precipitation in the city and its neighboring areas is the result of the combined effect of regional climate background conditions and urbanization process. Analyzing the relationship between urban and rural stations can strip the impact of climate change on extreme precipitation and determine the law of impact of urbanization on extreme precipitation. The process of urbanization can also be 

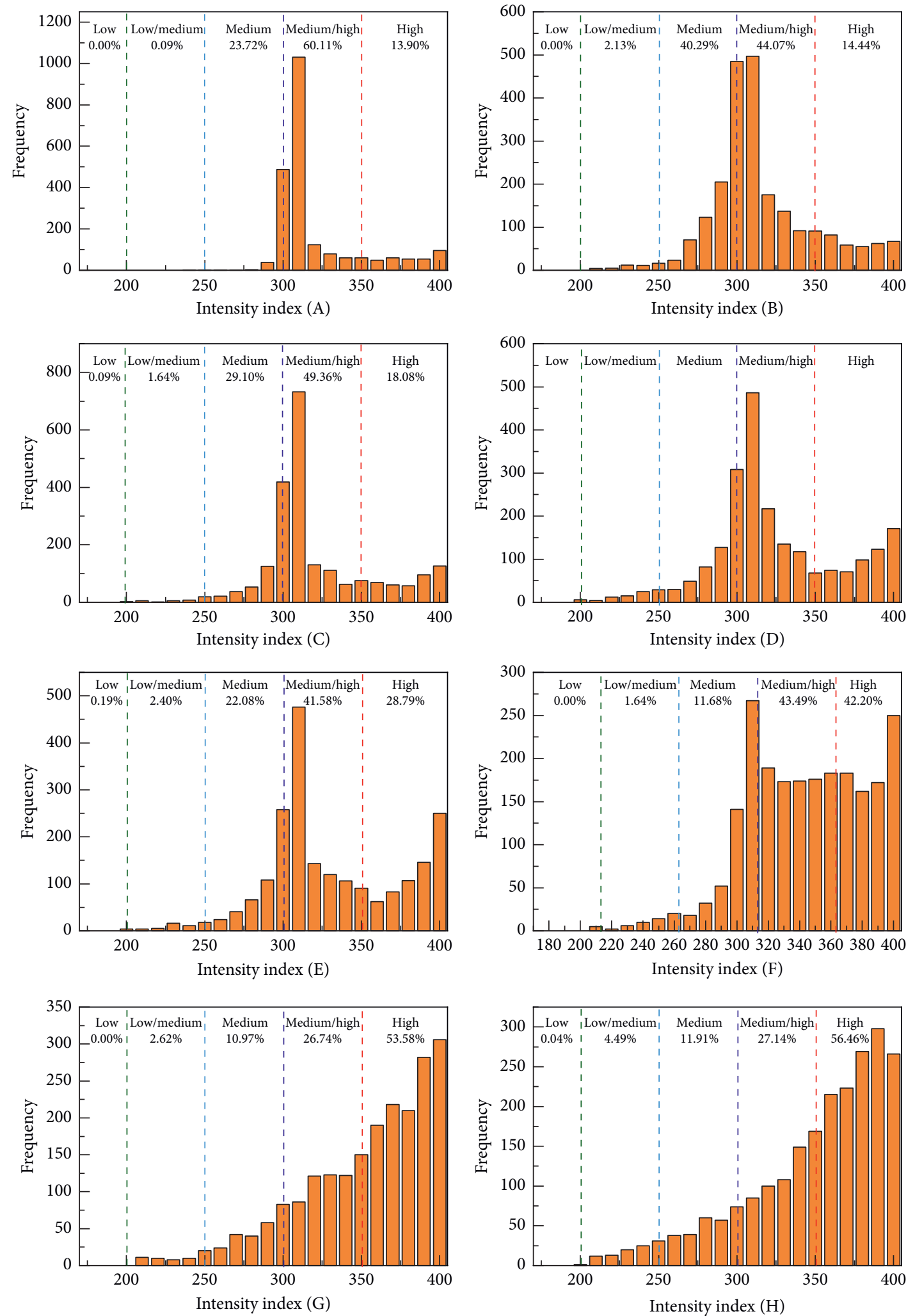

Figure 8: Histograms of frequency counts of land use intensities in the central urban area of the study area from 1984 to 2018. 

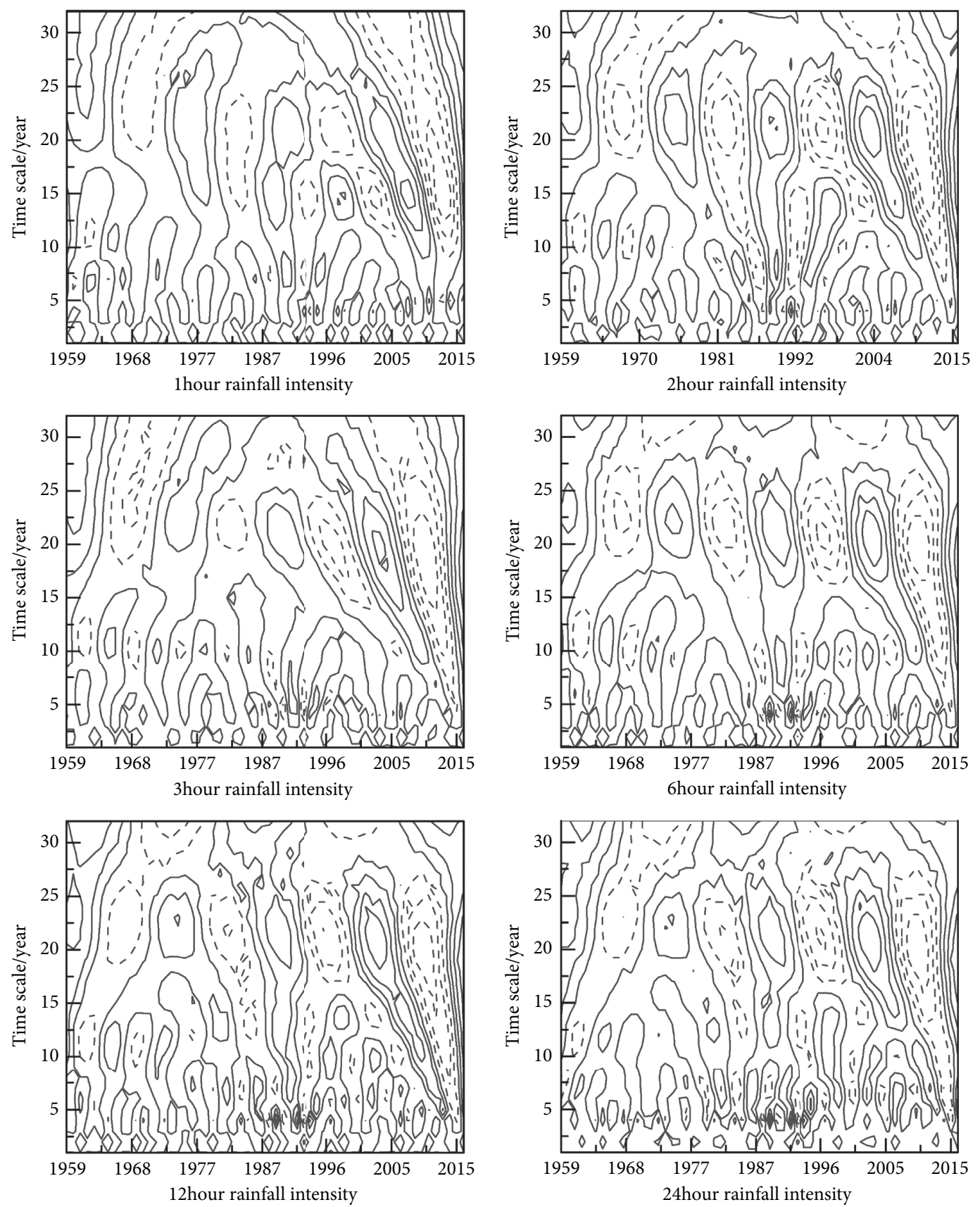

FIGURE 9: Contour plots of the real parts of the wavelet coefficients of the intensities of the precipitation with different durations in the study area from 1959 to 2018 .

characterized by changes in urban building area. Therefore, in order to discuss the impact of urbanization on rainfall, on the one hand, we analyzed the characteristics of long-term rainfall changes in urban and rural stations; on the other hand, we analyzed the changes in urban building area and rainfall trends.

5.3.1. Analysis of Precipitation Changes in Urban and Rural Stations. The MK nonparametric statistical test is conducted to obtain the variations in the intensities and frequencies of extreme precipitation with different durations at station S2
(Table 4). The results show the following. There are differences between the variation trends of the intensities of extreme precipitation with different durations at station S2. In particular, $\mathrm{Rx} 1 \mathrm{~h}$ and $\mathrm{Rx} 3 \mathrm{~h}$ exhibit insignificant decreasing trends and low confidence levels, with decreases of -0.007 and $-0.03 \mathrm{~mm} / 10 \mathrm{a}$, respectively. $\mathrm{Rx} 2 \mathrm{~h}, \mathrm{Rx} 6 \mathrm{~h}, \mathrm{Rx} 12 \mathrm{~h}$, and $\mathrm{Rx} 24 \mathrm{~h}$ show insignificant increasing trends and low confidence levels, with increases of $0.003,0.01,0.15$, and $0.05 \mathrm{~mm} /$ $10 \mathrm{a}$, respectively. In addition, the number of days of precipitation above the level of heavy rain has a $Z$-score of zero and has a variation trend. The number of days of rainfall above the level of rainstorm and the number of hours of 


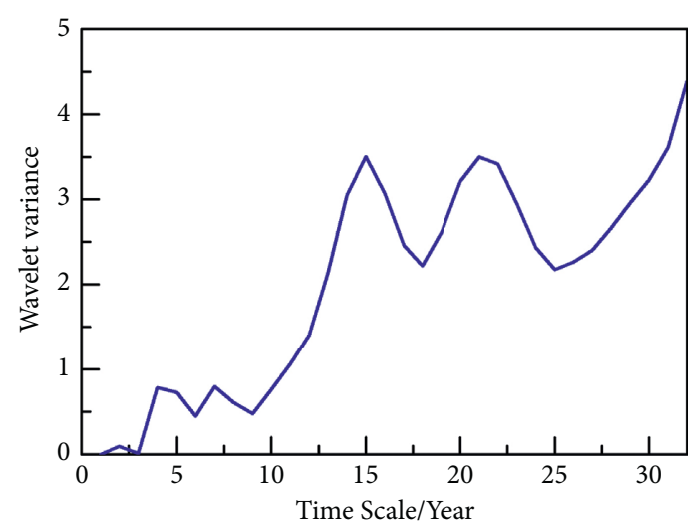

_ 1hour precipitation intensity wavelet variance curve

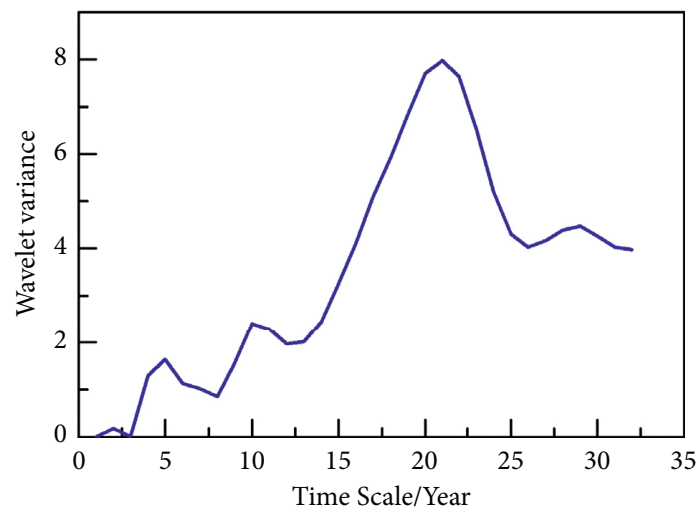

_ 3hour precipitation intensity wavelet variance curve

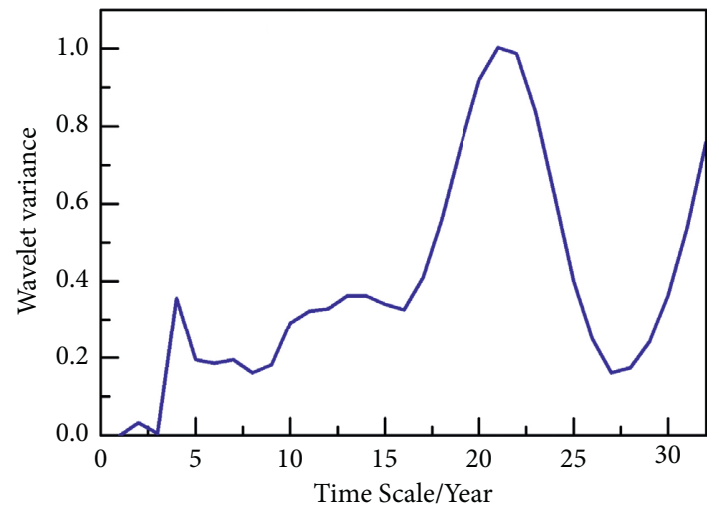

— 12hour precipitation intensity wavelet variance curve

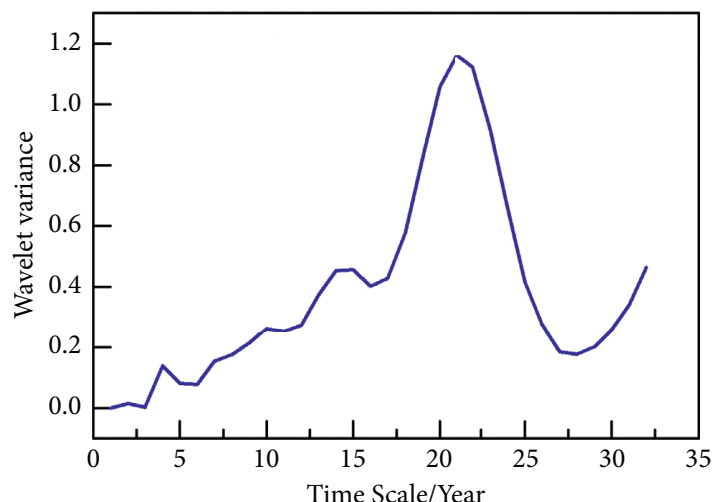

_ 2hour precipitation intensity wavelet variance curve

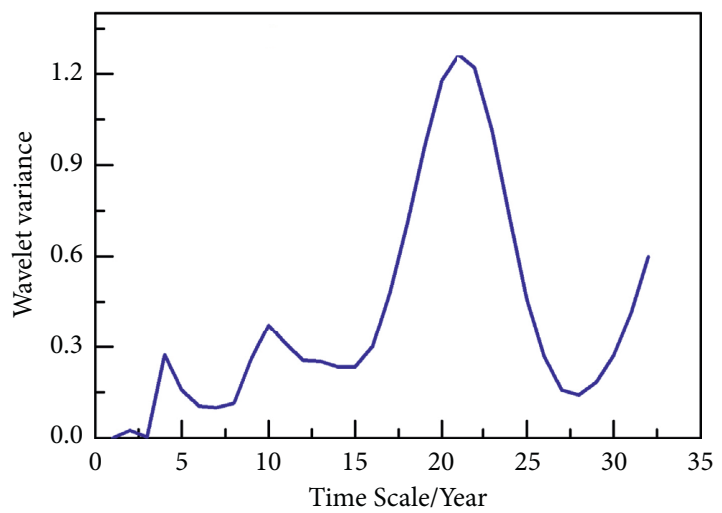

_ 6hour precipitation intensity wavelet variance curve

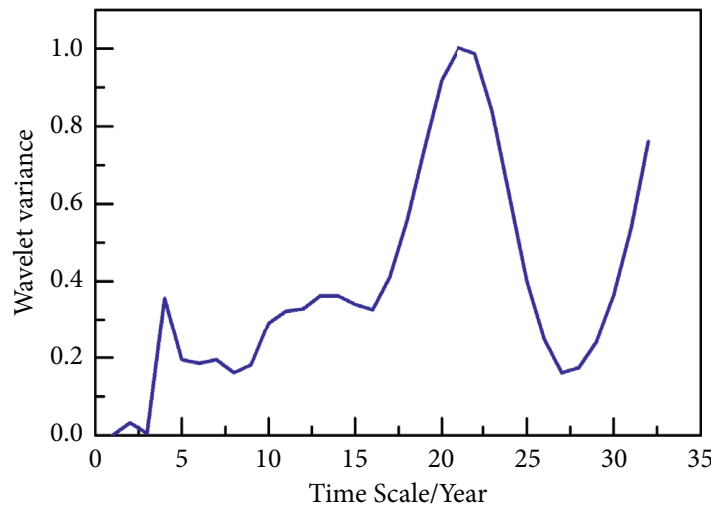

_ 24hour precipitation intensity wavelet variance curve

FIgURE 10: Plots of the wavelet variances of the intensities of precipitation with different durations in the study area from 1959 to 2018.

extreme precipitation exhibit an insignificant increasing trend and a low confidence level, and no variation is detected using the nonparametric Sen's method. That is, the variation trends of the intensities and frequencies of the extreme precipitation at station S2 are insignificant under natural conditions.

The double accumulative curve method is used to analyze the variations in the intensities of the precipitation with different durations at stations S1 and S2 from 1982 to 2018
(Figure 12). The results show that the inflection points of the slopes of the double cumulative curves of Rx1h and $\mathrm{Rx} 2 \mathrm{~h}$ both appear in 1989. Prior to 1989, the intensities of the extreme precipitation at the urban and rural stations were basically consistent. After 1989, the extreme precipitation at the urban station became higher than that at the rural station. There is minimal variation in the slopes of the double cumulative curves of the other extreme precipitation intensity indices, indicating that the urbanization in the 

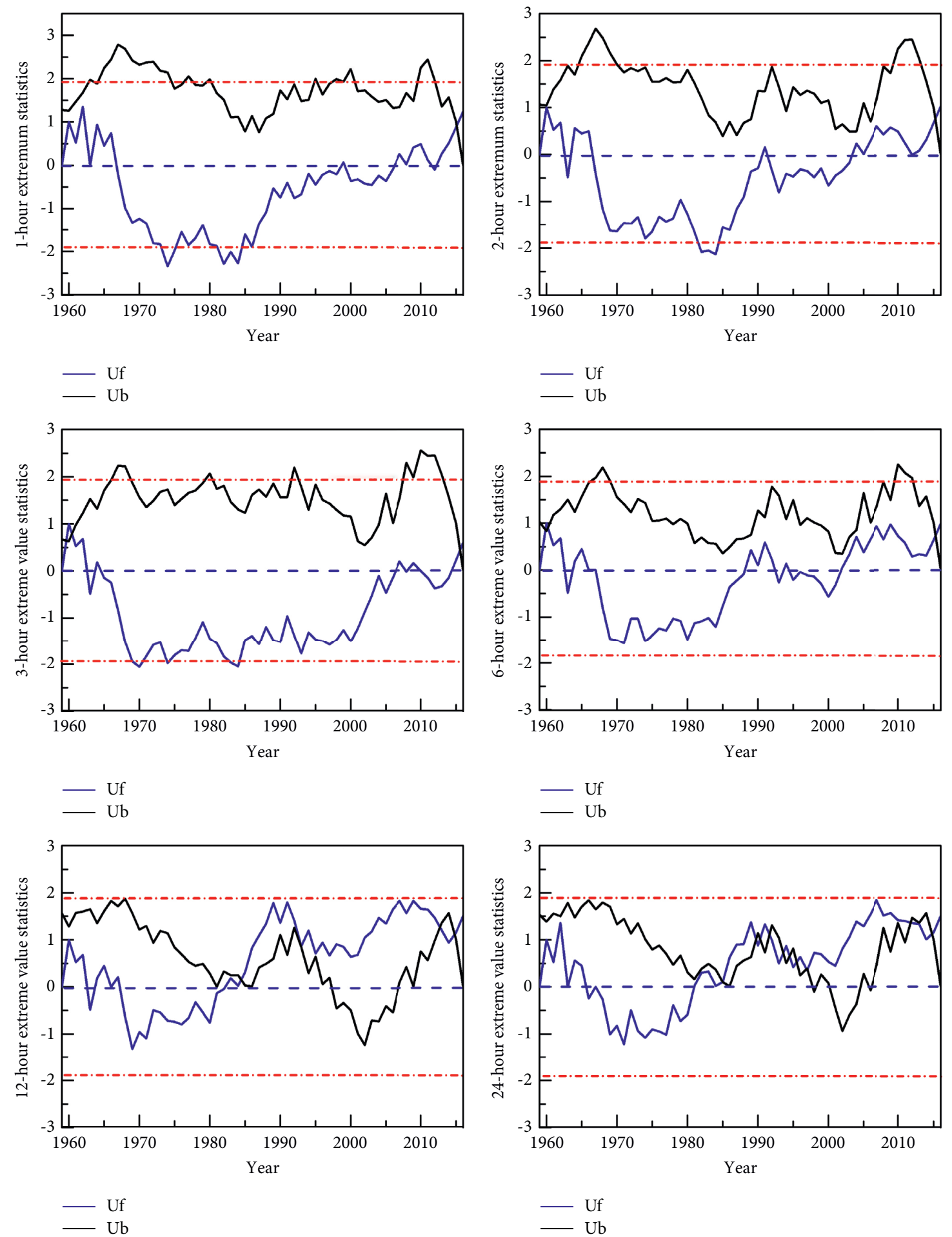

FIgURE 11: Abrupt changes in intensities of precipitation with different durations in the study area from 1959 to 2018.

study area has a large impact on short-duration precipitation but minimal impact on long-duration precipitation.

To further clarify the differences in the extreme precipitation before and after urbanization, the year of 1989 is used as a node to divide the series of extreme precipitation in the study area into two time periods, namely, 1959 to 1989 and 1989 to 2018 , based on which the variations in the means, variances, and maximums of $\mathrm{Rx} 1 \mathrm{~h}$ and $\mathrm{Rx} 2 \mathrm{~h}$ are statistically analyzed (Table 5). The statistical indices of Rx1h at the Xi'an station after 1989 are all higher than those in the former time period, specifically, the mean, variance, and range of $\mathrm{Rx} 1 \mathrm{~h}$ in the latter time period are $7.09 \mathrm{~mm} / \mathrm{h}$, $5.74 \mathrm{~mm} / \mathrm{h}$, and $27.3 \mathrm{~mm} / \mathrm{h}$, respectively, higher than those in the former time period. The mean, variance, and range of 
TABle 4: MK tests and slopes of indices of extreme precipitation in the rural area of the study area.

\begin{tabular}{lccc}
\hline Extreme precipitation index & Z-score & Variation magnitude of nonparametric Sen's & Confidence level (\%) \\
\hline Rx1h & -0.5 & $-0.07(\mathrm{~mm} / 10 \mathrm{a})$ & $<90$ \\
Rx2h & 0.25 & $0.03(\mathrm{~mm} / 10 \mathrm{a})$ & $<90$ \\
Rx3h & -0.14 & $-0.03(\mathrm{~mm} / 10 \mathrm{a})$ & $<90$ \\
Rx6h & 0.04 & $0.01(\mathrm{~mm} / 10 \mathrm{a})$ & $<90$ \\
Rx12h & 0.36 & $0.15(\mathrm{~mm} / 10 \mathrm{a})$ & $<90$ \\
Rx24h & 0.23 & $0.05(\mathrm{~mm} / 10 \mathrm{a})$ & $<90$ \\
R25mm & 0 & $0(\mathrm{~d} / 10 \mathrm{a})$ & $<90$ \\
R50mm & 0.18 & $0(\mathrm{~d} / 10 \mathrm{a})$ & $<90$ \\
R99p & 0.47 & $0(\mathrm{~d} / 10 \mathrm{a})$ & $<90$ \\
\hline
\end{tabular}
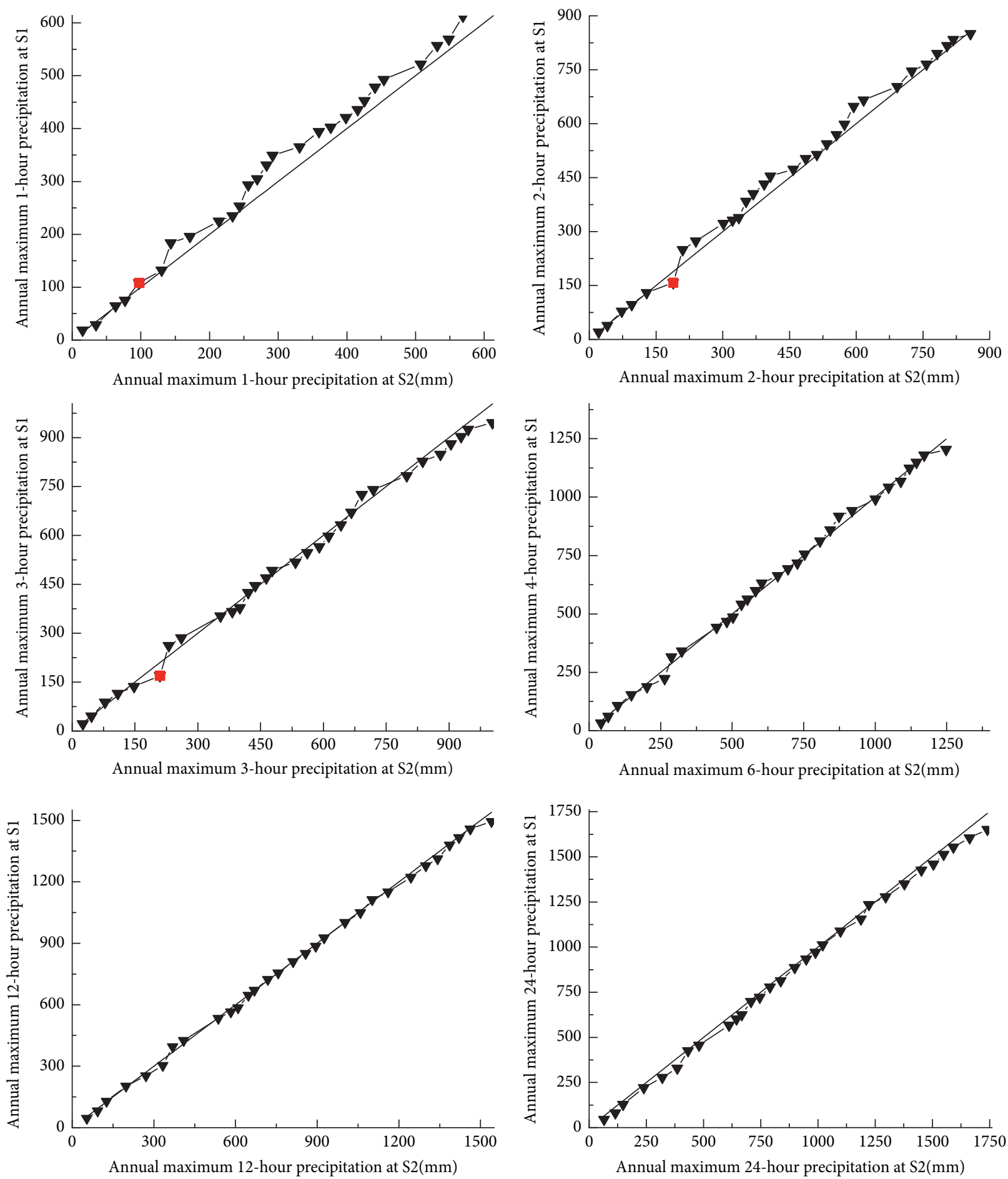

FIgURE 12: Double cumulative curves of the precipitation at urban and rural stations of the study area from 1982 to 2018. 
TABle 5: Statistical indices of extreme precipitation before and after urbanization in the study area.

\begin{tabular}{lcccc}
\hline Statistical index & Rx1h & & \multicolumn{2}{c}{ Rx2h } \\
& $1959-1989$ & $1989-2018$ & $1959-1989$ & 31.82 \\
Mean & 18.38 & 25.47 & 24.05 & 9.63 \\
Variance & 8.3 & 14.04 & 49.1 & 9.5 \\
Maximum & 35.9 & 63.2 & 92.4 \\
\hline
\end{tabular}

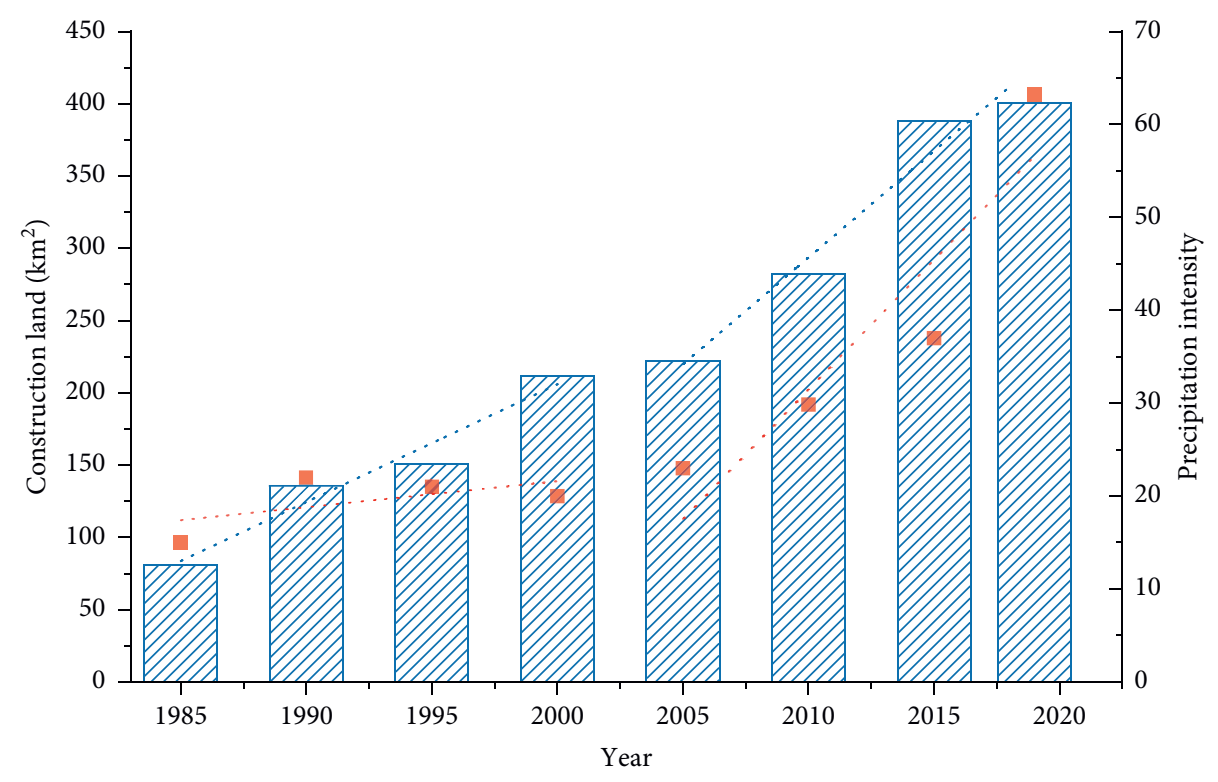

TII Construction land

- Precipitation intensity

FIGURE 13: Construction land and precipitation intensity trend in the study area.

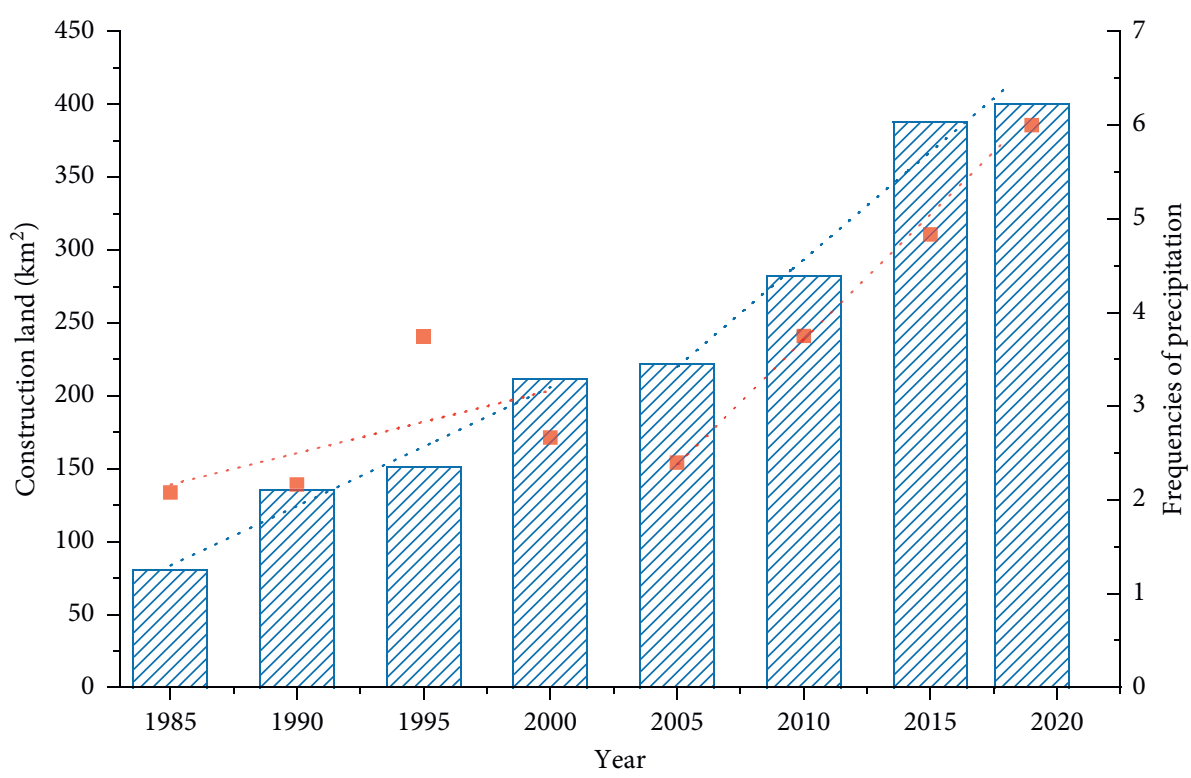

Construction land

- Frequencies of precipitation

Figure 14: Construction land and frequencies of precipitation trend in the study area. 
$\mathrm{Rx} 2 \mathrm{~h}$ at the $\mathrm{Xi}$ 'an station in the latter time period are $7.75 \mathrm{~mm} / \mathrm{h}, 9.87 \mathrm{~mm} / \mathrm{h}$, and $43.2 \mathrm{~mm} / \mathrm{h}$ higher than those in the former time period. Thus, the variances and ranges of both $\mathrm{Rx} 1 \mathrm{~h}$ and $\mathrm{Rx} 2 \mathrm{~h}$ increase after urbanization, and the increase in the maximum of the extreme precipitation intensities leads to an increase in the peak values in the time series with increasing variation magnitudes, thus resulting in an increase in the variances of the extreme precipitation series as well as in the fluctuation magnitude.

5.3.2. Test of the Differences between the Precipitation at the Representative Urban and Rural Stations. The impact of urbanization on rainfall is characterized by the trend of urban area and rainfall characteristics (Figures 13 and 14). From the 1980s to the present, the construction area, rainfall intensity, and frequency of rainfall above $10 \mathrm{~mm}$ in the study area have all shown an increasing trend. From 1980s to 2000 s, the increase in rainfall frequency and rainfall intensity was less than the growth trend of building area; after 2000s, the rainfall frequency, rainfall intensity, and building area all increased rapidly, and the growth trends of the three tended to be consistent.

\section{Conclusion}

In this study, we analyzed the patterns of land use and the characteristics of extreme precipitation during the urbanization process, using the city of Xi'an in China as a study area. On this basis, we discussed the response of urbanization to extreme precipitation. The main conclusions are summarized as follows:

(1) During the urbanization of the study area, the farmland area sharply decreased in size, the construction land area rapidly expanded, and the vegetation area increased and fluctuated. The transfer of land use in the central urban area occurred mainly as the transfer of farmland to other types of land use, while the transfers between other types of land use were not significant. The intensities of land use in the central urban area continuously increased.

(2) The intensities and frequency indices of the extreme precipitation with different durations in the study area all showed increasing trends. Specifically, the extreme precipitation intensities $\mathrm{Rx} 1 \mathrm{~h}, \mathrm{Rx} 12 \mathrm{~h}$, and $\mathrm{Rx} 24 \mathrm{~h}$ all passed the significance test, with magnitude increases of $1.06,1.64$, and $2.16 \mathrm{~mm} / 10 \mathrm{a}$, respectively. During the flood season, the intensities of extreme precipitation with different durations all exhibited periodic alternations with a dominant period of 21 years. Both the magnitude and maximum value of the periodic variations increased with time. Abrupt changes could occur in the intensities of the precipitation with different durations. However, the significance of the abrupt change points was low, and the extreme precipitation series fluctuated greatly.
(3) The urbanization in the study area had a large impact on the Rx1h and Rx2h precipitation starting in 1989, while it had minimal impact on the precipitation with long duration. The mean, variance, and range of Rx1h after urbanization are $7.09 \mathrm{~mm} / \mathrm{h}, 5.74 \mathrm{~mm} / \mathrm{h}$, and $27.3 \mathrm{~mm} / \mathrm{h}$ higher, respectively, than those before urbanization. The mean, variance, and range of $\mathrm{Rx} 2 \mathrm{~h}$ are $7.75 \mathrm{~mm} / \mathrm{h}, 9.87 \mathrm{~mm} / \mathrm{h}$, and $43.2 \mathrm{~mm} / \mathrm{h}$ higher, respectively, in the former time period. Hence, urbanization has a significant amplification effect on the increase in the intensity of extreme precipitation. With the development of urbanization, regional rainfall intensity and rainfall frequency have increased rapidly. After the 2000s, the growth trend of urbanization process, rainfall intensity, and rainfall frequency is highly consistent.

\section{Data Availability}

The data used to support the findings of this study are available from the corresponding author upon request.

\section{Conflicts of Interest}

The authors declare that they have no conflicts of interest.

\section{Acknowledgments}

This work was supported by the National Natural Science Foundation of China (project nos. 51979222 and 41807156) and the Water Science and Technology Foundation of Shaanxi Province of China (no. 2017slkj-13).

\section{References}

[1] World Urbanization Prospects, United Nations Department of Economic and Social Affairs, 2019.

[2] N. Harman, The Energy Balance of Urban Areas, University of Reading, Reading, UK, 2003.

[3] J. Cheng, Effects of Land Use and Land Cover Change on Environmental Hydrology in the Center Urban Area of Shanghai, East China Normal University, Shanghai, China, 2007.

[4] S. Grimmond, "Urbanization and global environmental change: local effects of urban warming," The Geographical Journal, vol. 173, no. 1, pp. 83-88, 2007.

[5] W. Nie, B. F. Zaitchik, G. Ni, and T. Sun, "Impacts of anthropogenic heat on summertime rainfall in Beijing," Journal of Hydrometeorology, vol. 18, no. 3, pp. 693-712, 2017.

[6] R. Bornstein and Q. Lin, "Urban heat islands and summertime convective thunderstorms in Atlanta: three case studies," Atmospheric Environment, vol. 34, no. 3, pp. 507-516, 2000.

[7] S. Miao, F. Chen, M. A. LeMone, M. Tewari, Q. Li, and Y. Wang, "An observational and modeling study of characteristics of urban heat island and boundary layer structures in Beijing," Journal of Applied Meteorology and Climatology, vol. 48, no. 3, pp. 484-501, 2009.

[8] S. A. Changnon, "The La Porte weather anomaly-fact or fiction?" Bulletin of the American Meteorological Society, vol. 49, no. 1, pp. 4-11, 1968.

[9] X. Li, S. Ning, P. Zhang, and W. Yang, "Environmental pollution and health risks of heavy metals in the soil around a 
construction waste landfill," International Journal of Design \& Nature and Ecodynamics, vol. 15, no. 3, pp. 393-399, 2020.

[10] L. M. Hand and J. M. Shepherd, "An investigation of warmseason spatial rainfall variability in Oklahoma city: possible linkages to urbanization and prevailing wind," Journal of Applied Meteorology and Climatology, vol. 48, no. 2, pp. 251-269, 2009.

[11] J. J. Baik, Y. H. Kim, J. J. Kim, and J. Y. Han, "Effects of boundary-layer stability on urban heat island-induced circulation," Theoretical and Applied Climatology, vol. 89, no. 1, pp. 73-81, 2007.

[12] S. Pfahl, P. A. O'Gorman, and E. M. Fischer, "Understanding the regional pattern of projected future changes in extreme precipitation," Nature Climate Change, vol. 7, no. 6, pp. 423-427, 2017.

[13] J.-L. Fan, Y.-J. Zhang, and B. Wang, "The impact of urbanization on residential energy consumption in China: an aggregated and disaggregated analysis," Renewable and Sustainable Energy Reviews, vol. 75, pp. 220-233, 2017.

[14] L. Shi, H. Taubenböck, Z. Zhang, F. Liu, and M. Wurm, "Urbanization in China from the end of 1980s until 2010spatial dynamics and patterns of growth using EO-data," International Journal of Digital Earth, vol. 12, no. 1, pp. 78-94, 2019.

[15] M. O. Andreae, D. Rosenfeld, P. Artaxo et al., "Smoking rain clouds over the Amazon," Science, vol. 303, no. 5662, pp. 1337-1342, 2004.

[16] Y. J. Kaufman and R. S. Fraser, "The effect of smoke particles on clouds and climate forcing," Science, vol. 277, no. 5332, pp. 1636-1639, 1997.

[17] F. Chabane, A. Arif, and S. Benramache, "The estimate of aerosol optical depth for diverse meteorological conditions," Instrumentation, Measure, Métrologie, vol. 19, no. 2, pp. 119-123, 2020.

[18] D. Rosenfeld, "Suppression of rain and snow by urban and industrial air pollution," Science, vol. 287, no. 5459, pp. 1793-1796, 2000.

[19] Q. Hu, J. Y. Zhang, Y. T. Wang, Y. Huang, Y. Liu, and L. J. Li, "A review of urbanization impact on precipitation," Advances in Water Resources, vol. 29, pp. 138-150, 2018.

[20] H. Lin and J. N. Sun, "Possible effects of urbanization on regional precipitation over Yangtze river delta area," National Sciences, vol. 50, pp. 792-799, 2014.

[21] Y. R. Jin, Q. F. Hu, Y. T. Wang, Y. Huang, H. B. Yang, and T. T. Cui, "Impacts of rapid urbanization on precipitation at two representative rain gauges in Shanghai city," Journal of Hohai University National Sciences, vol. 45, pp. 204-210, 2017.

[22] S.-H. Lee, S.-W. Kim, W. M. Angevine et al., "Evaluation of urban surface parameterizations in the WRF model using measurements during the Texas air quality study 2006 field campaign," Atmospheric Chemistry and Physics, vol. 11, no. 5, pp. 2127-2143, 2011.

[23] X. Wang, J. Liao, J. Zhang et al., "A numeric study of regional climate change induced by urban expansion in the Pearl river delta, China," Journal of Applied Meteorology and Climatology, vol. 53, no. 2, pp. 346-362, 2014.

[24] H. Zhang, C. Wu, W. Chen, and G. Huang, "Effect of urban expansion on summer rainfall in the pearl river delta, south China," Journal of Hydrology, vol. 568, pp. 747-757, 2019.

[25] D. Wu, "Rainstorm water-logging origin analysis and simulation study of Xi'an typical area," M.S. thesis, Chang'an University, Xi'an, China, 2015.

[26] X. M. Song, J. Y. Zhang, G. Q. Wang, R. M. He, and X. J. Wang, "Development and challenges of urban hydrology in a changing environment: II: Urban stormwater modeling and management," Advances in Water Science, vol. 25, no. 5, pp. 752-764, 2014.

[27] H. Cui and F. Gao, "Spatiotemporal distribution of air pollutants and process of severe air pollution: evidence from a highly polluted industrial and mining city," International Journal of Sustainable Development and Planning, vol. 15, no. 3, pp. 385-391, 2019.

[28] P. Liu, Z. Fang, C. Lv, and A. Ruan, "China's agricultural water-use efficiency and its influencing factors under the constraint of pollution emission," International Journal of Design \& Nature and Ecodynamics, vol. 15, no. 4, pp. 579-585, 2020.

[29] Y. Xin, "Characteristic analysis of change in precipitation frequency in Xi'an city," Journal of Natural Disasters, vol. 20, no. 4, pp. 60-65, 2011.

[30] Z. Zhou, N. Hu, and H. Zhou, "Analysis on the change trend of temperature and precipitation in Xi' an during the period of 1961-2009," Gan Han Qu Yan Jiu, vol. 29, no. 1, pp. 27-34, 2012.

[31] D. Zhuang and J. Liu, "Study on the model of regional differentiation of land use degree in China," Journal of Natural Resources, vol. 12, no. 2, pp. 105-111, 1997. 